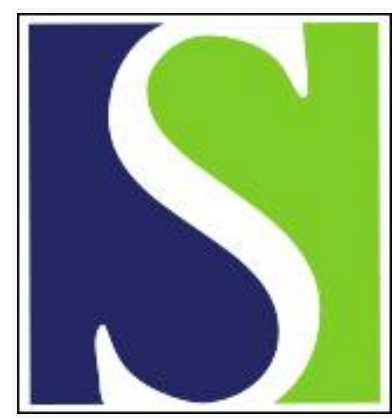

Scand J Work Environ Health 2007;33(6):454-464

https://doi.org/10.5271/sjweh.1169

Issue date: 31 Dec 2007

Male bladder cancer risk and occupational exposure according to a job-exposure matrix-a case-control study in British Columbia, Canada

by Richardson K, Band PR, Astrakianakis G, Le ND

Affiliation: Cancer Control Research, BC Cancer Research Centre, 675 West 10th Avenue, Vancouver, BC, Canada V5Z 1 L3. nle@bccrc.ca

Refers to the following text of the Journal: 2004;30(5):350-355

Key terms: automobile exhaust; bladder cancer; Canada; case-control study; construction; detergent; job-exposure matrix; logging industry; lubricating oil; male; occupational exposure; petroleum additive; petroleum product; solvent

This article in PubMed: www.ncbi.nlm.nih.gov/pubmed/18327514 


\title{
Male bladder cancer risk and occupational exposure according to a job- exposure matrix - a case-control study in British Columbia, Canada
}

\author{
by Kathryn Richardson, MSc, , 2 Pierre R Band, MD,1, 3 George Astrakianakis, PhD, 1, 4 Nhu D Le, PhD 1, 2
}

\begin{abstract}
Richardson K, Band PR, Astrakianakis G, Le ND. Male bladder cancer risk and occupational exposure according to a job-exposure matrix - a case-control study in British Columbia, Canada. Scand J Work Environ Health. 2007;33(6):454-464.
\end{abstract}

\begin{abstract}
Objectives The authors investigated the risk of bladder cancer in association with exposure to over $12000 \mathrm{oc}-$ cupational chemical agents, complex mixtures, and other substances (hereafter referred to as chemical agents). Methods Adult males diagnosed with cancer between 1983 and 1990 in British Columbia, Canada, were surveyed. Detailed occupational histories and confounding information was provided by a self-administered questionnaire. Cancer controls were matched to bladder cancer cases, resulting in 1062 cases and 8057 controls for the analysis. An extensive United-States-based job-exposure matrix was applied to estimate cumulative exposure to occupational chemical agents. Odds ratios for bladder cancer due to exposure to chemical agents were estimated via conditional logistic regression analyses, adjusted for important confounders.

Results A significantly $(\mathrm{P}<0.05)$ increased risk was detected for ever exposure to 635 chemical agents, and 341 chemical agents exhibited a significantly increasing dose-response relationship. Adjustment for multiple comparisons resulted in a subset of 29 chemical agents that continued to show significant results. A principal components analysis classified these 29 chemical agents into five independent groups, distinguished mainly by job. Exposures to these chemical agents were largely due to employment in the logging and construction industries and occupations involving motor vehicles. Consistent results were observed for bladder carcinogens reported in the literature.

Conclusions This study suggests that several specific chemical agents were significantly associated with the risk of bladder cancer. The chemical agents were mainly derivatives or combustion products of fossil fuels. The results corroborate important findings from the literature and document a risk for specific chemical agents not previously reported.
\end{abstract}

Key terms automobile exhaust; construction; detergent; logging industry; lubricating oil; petroleum additive; petroleum product; solvent.

The incidence of bladder cancer is greatest for Caucasian males in developed countries (1). An estimated 5000 and 1700 cases will be diagnosed for men and women, respectively, in Canada during 2007, bladder cancer therefore being the fourth most commonly diagnosed cancer among men (2). Cigarette smoking is the main risk factor for bladder cancer, and it accounts for around half of all cases among men (1). Currently the role of other lifestyle and dietary factors is unclear, due to a lack of studies adjusting for the many potential confounders and consistently showing a strong causal link $(1,3)$.
The second most important risk factor is occupation, which is estimated to be responsible for up to $24 \%$ of all bladder cancers (4). Occupational exposure to the following chemical agents is associated with an increased risk of bladder cancer: aromatic amines, paints and solvents, dyes, leather dust, polycyclic aromatic hydrocarbons (PAH), motor exhaust, and chlorinated hydrocarbons $(1,5-8)$. Further epidemiologic research is required for many other potential bladder carcinogens.

Excess bladder cancer incidence was previously observed within specific occupations and industries in a case-control study in British Columbia, Canada (9).

1 Cancer Control Research, BC Cancer Research Centre, Vancouver, British Columbia, Canada.

2 University of British Columbia, Vancouver, British Columbia, Canada.

3 Division of Biostatistics and Epidemiology, Environmental Health Sciences Bureau, Healthy Environments and Consumer Safety Branch, Health Protection Branch, Health Canada, Ottawa, Ontario, Canada.

4 Occupational Health and Safety Agency for Health Care in BC, Vancouver, British Columbia, Canada.

Reprint requests to: Dr Nhu D Le, Cancer Control Research, BC Cancer Research Centre, 675 West 10th Avenue, Vancouver, BC, Canada V5Z 1L3. [E-mail: nle@bccrc.ca] 
Using the same cancer register-based dataset, we investigated which specific chemical agents may be responsible for the excess risks observed. Cumulative exposures to 12456 different occupational chemical agents were estimated with the use of a job-exposure matrix from the United States (US) and the participants' job histories. This article summarizes the findings, including results for the substances classified by the International Agency for Cancer Research (IARC) as definite or probable carcinogens for bladder cancer $(6,7)$.

\section{Study population and methods}

\section{Study population}

The study design has been described elsewhere $(9,10)$ and is summarized here. A self-administered questionnaire was mailed to male cancer patients ascertained by the British Columbia Cancer Registry, aged 20 years and older at the time of diagnosis, during the period 1983 to 1990. Although occupational bladder cancer has been suspected for women, only data for men were available for this analysis. The questionnaire requested information on sociodemographic factors such as ethnicity, education, marital status, and lifetime smoking and drinking habits. Detailed lifetime job descriptions were requested for all jobs of at least 1 year in duration, including the location and duration (years) of each job, and whether the job was full-time or part-time.

Questionnaires were sent to 25726 eligible men, and 15463 forms were returned (60.1\%). Of the respondents, 1129 had bladder cancer. Histological confirmation of the diagnosis was obtained for all of the cases. The anatomic tumor site was coded using the ninth revision of the International Classification of Diseases (11) and grouped into three-digit categories for the analysis. Job descriptions were coded according to the four-digit 1980 Canadian Standard Industrial Classification (CSIC) and the 1980 Standard Occupational Classification (CSOC) codes $(12,13)$.

\section{Exposure assessment}

Exposure to various occupational substances was assessed with the use of data from the National Occupational Exposure Survey (NOES) that was based on the job-exposure matrix (JEM) developed by the United States (US) National Institute for Occupational Safety and Health $(14,15)$. The NOES-JEM is essentially a three-dimensional array with US industry codes on one axis, US occupational codes on another axis, and five-digit substance codes on a third axis. The elements of the array indicate the probability of exposure to a specific substance in a specific job. As calendar year is not included in the matrix, the probability of exposure is independent of time. Jobs were classified according to the US 1980 census of the population industrial (USCENIND) and occupational (USCENOCC) classifications, which consist of 231 industrial and 503 occupational three-digit codes (16).

The NOES involved walk-through assessments carried out by trained engineers in a stratified sample of 4490 workplaces within the United States during 1981 to 1983. The number of workers exposed to over 12000 chemical, physical, and biological agents employed within 121 industrial and 377 occupational categories was recorded. Exposure was defined as that persisting beyond 30 minutes per workweek or occurring at least once per week for $90 \%$ of the weeks of the work year. Some industries were excluded by NOES due to few expected exposures (eg, financial, insurance, and real estate facilities). We assumed these industries were unexposed.

Comparison of Canadian job exposures via the US NOES-JEM was facilitated by a linkage system previously developed by experts in occupational coding that translated the 860 CSIC codes to USCENIND equivalents and the 503 CSOC codes to USCENOCC equivalents (17). The relationship between the Canadian and US codes was often not one-to-one. For example, CSOC 8562 upholsterers translates to two USCENOCC codes (668 upholsterers and 785 assemblers). Eighty-four percent of the Canadian industries had exactly one US translation, and $16 \%$ had more than one US translation with a maximum of seven translations. Forty-two percent of the Canadian occupations had one US translation, and $58 \%$ had more than one US translation with a maximum of 31 translations. We considered each translation for a particular Canadian occupation or industry equally applicable. Thus the probability of exposure in a given $\mathrm{Ca}$ nadian job was obtained by first translating the Canadian industry and occupation codes into US equivalents and then averaging the exposure probabilities obtained from the NOES-JEM for all possible translated US industry and occupation combinations. We assumed exposure was zero for translated US industry-occupation combinations not found in the JEM.

\section{Cumulative exposure}

Cumulative exposure (expressed as expected work years of exposure) to each chemical agent was calculated as the product of the probability of exposure and job duration (in work years), summed across all recorded Canadian jobs. A work year was defined as 1 year in a full-time job or 2 years in a part-time job. All job histories were truncated at 5 years prior to diagnosis to account for possible latency periods. 


\section{Data analysis}

The controls comprised internal controls of all other cancer sites, excluding lung cancer and cancers of an unknown primary site. Completed questionnaires were available for 1129 possible bladder cases, mostly transitional cell carcinoma (94\%), and 10628 eligible controls. The reliability of the information on occupational histories has been examined previously and was reported to be very good (9). A further 63 cases (5.6\%) and 453 controls (4.3\%) were excluded due to incomplete occupational histories. Controls were matched to the cases according to year of birth and year of diagnosis. Matching resulted in 1062 bladder cancer cases and 8057 cancer controls for the analysis. The median number of matched controls per case was 7 .

We excluded the biological and physical agents and restricted our analysis to the remaining 12456 chemical agents covered by the job-exposure matrix. The risk of developing bladder cancer due to cumulative exposure was analyzed using a conditional logistic regression (18, 19). The risk was also adjusted for potentially important confounders that were identified by entering all possible confounders into a model and taking a backwards stepwise approach retaining all variables with a P-value of $<0.2$. The following variables were considered to be possible confounders: ethnic origin, marital status, education, questionnaire respondent, alcohol consumption, and cigarette smoking status (eg, ever versus never, years since quitting, cigarettes per day, smoking duration, pack-years).

As nonlinear associations between the exposures and risk were anticipated, the cumulative exposure variable for each chemical agent was transformed to an ordinal scale with four categories. The first category was for no exposure. The nonzero cumulative exposures were split into tertiles according to the exposed controls. The categories were labeled "unexposed", "low", "medium", and "high" exposure and were coded as scores of zero, one, two, and three to construct an ordinal variable. Adjusted odds ratios (OR) and corresponding confidence intervals (CI) were estimated for ever versus never exposure for the chemical agents with at least three cases ever exposed. OR values with a P-value of $<0.05$ (two-sided test) were considered statistically significant; hereafter "significant" means "statistical significance" for brevity.

Chemical agents with at least three exposed cases were selected to be analyzed in this report. There is always a trade-off for such a choice. Choosing those with a large number of exposed cases would lead to more-precise estimates with less chance of false positives; on the other hand, important associations with a smaller number of exposed cases would be missed. Using a criterion with a small number of exposed cases would allow for opportunities to identify all potential associations, even those with only a few exposed cases. However, this approach would lead to less-precise estimates and a higher chance for false positives. The choice of at least 3 exposed cases is often used in epidemiology studies for hypothesis-generating objectives. This is an aim of this study since not many studies have reported on risks based on chemical agents. Furthermore, in principle, users can use the information provided in the appendix (placed on the homepage of the Scandinavian Journal of Work Environment \& Health) to derive results corresponding with any higher cut-off point for the number of exposed cases.

Dose-response relationships for chemical agents with at least nine exposed cases were examined. The OR values and corresponding confidence intervals were estimated for low, medium, and high versus zero exposure. A linear trend test was considered by treating the cumulative exposure on the transformed ordinal scale as a continuous variable.

As many chemical agents and hence hypotheses were tested, multiple comparisons were accounted for by controlling the false discovery rate (FDR) to below 5\% according to Benjamini \& Hochberg (20). This FDR method controls the level of false positives expected among the selected chemical agents; that is, a 5\% false discovery rate means that it is expected that $95 \%$ of the selected chemical agents have been correctly identified. This method is less conservative than alternative methods that control the probability of at least one false positive, such as the Bonferroni method. The chosen method is intuitively more appealing in our study since it looks at the type-I error rate in the list of chemical agents selected (as opposed to the probability of any type-I error-commonly referred to as the P-value), and has greater power than other procedures.

Chemical agents that had at least nine cases exposed and that, after adjustment for multiple comparisons, either exhibited a significantly increased OR value for ever exposure or a significantly increasing linear trend test were considered as potentially exhibiting bladder carcinogenic properties. These chemical agents were selected for further analysis.

High correlations between the cumulative exposures of some of the selected chemical agents were expected, as similar levels of exposure with different agents could occur within the same jobs. A principal component analysis with Varimax rotation was performed on the ordinal cumulative exposure variables to examine the interrelations among the selected chemical agents (21). Chemical agents were assigned to the principal components with which they had component loadings greater than 0.4 .

In addition, results are presented for chemical agents classified by IARC as definite or probable carcinogens for bladder cancer $(6,7)$. 


\section{Results}

Matching resulted in 1062 bladder cancer cases and 8057 cancer controls for the analysis. The controls were diagnosed with cancer at a range of primary sites, the most common being prostate (17.4\%), nonmelanoma skin (13.2\%), and colon (13\%). Cases and controls were comparable with regard to age, work years employed, education, and marital status (table 1). However, the bladder cases were more likely to be Caucasian, to have

Table 1. Selected characteristics of the case and control groups in a bladder cancer study, British Columbia, Canada, 1983-1990.

\begin{tabular}{|c|c|c|c|c|c|c|c|c|}
\hline \multirow[t]{2}{*}{ Characteristic } & \multicolumn{4}{|c|}{ Cases (N=1062) } & \multicolumn{4}{|c|}{ Controls ( $\mathrm{N}=8057)$} \\
\hline & N & $\%$ & Mean & SD & N & $\%$ & Mean & $\mathrm{SD}$ \\
\hline Age at diagnosis (years) & . & & 67.0 & 11.4 & . & & 65.9 & 10.9 \\
\hline Work years employed a & . & & 36.7 & 11.0 & . & & 35.9 & 11.2 \\
\hline \multicolumn{9}{|l|}{ Year of diagnosis } \\
\hline $\begin{array}{l}1983 \\
1984 \\
1985 \\
1986 \\
1987\end{array}$ & $\begin{array}{l}222 \\
215 \\
221 \\
216 \\
188\end{array}$ & $\begin{array}{l}20.9 \\
20.2 \\
20.8 \\
20.3 \\
17.7\end{array}$ & $\begin{array}{l}\dot{ } \\
\dot{.} \\
\dot{ }\end{array}$ & & $\begin{array}{l}2600 \\
1801 \\
1475 \\
1088 \\
1093\end{array}$ & $\begin{array}{l}32.3 \\
22.4 \\
18.3 \\
13.5 \\
13.6\end{array}$ & $\begin{array}{l}\cdot \\
\cdot \\
. \\
.\end{array}$ & 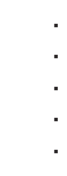 \\
\hline \multicolumn{9}{|l|}{ Ethnicity } \\
\hline $\begin{array}{l}\text { Caucasian } \\
\text { Non-Caucasian } \\
\text { Unknown }\end{array}$ & $\begin{array}{r}1027 \\
31 \\
4\end{array}$ & $\begin{array}{r}96.7 \\
2.9 \\
0.4\end{array}$ & . & . & $\begin{array}{r}7665 \\
350 \\
42\end{array}$ & $\begin{array}{r}95.1 \\
4.3 \\
0.5\end{array}$ & $\begin{array}{l}\cdot \\
\cdot\end{array}$ & 更 \\
\hline \multicolumn{9}{|l|}{ Level of education } \\
\hline $\begin{array}{l}\text { Grade } 11 \text { or lower } \\
\text { Grade } 12 \text { or higher } \\
\text { Unknown }\end{array}$ & $\begin{array}{r}598 \\
417 \\
47\end{array}$ & $\begin{array}{r}56.3 \\
39.3 \\
4.4\end{array}$ & : & . & $\begin{array}{r}4477 \\
3189 \\
391\end{array}$ & $\begin{array}{r}55.6 \\
39.6 \\
4.9\end{array}$ & . & 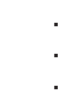 \\
\hline \multicolumn{9}{|l|}{ Marital status } \\
\hline $\begin{array}{l}\text { Married or cohabitating } \\
\text { Other } \\
\text { Unknown }\end{array}$ & $\begin{array}{r}889 \\
165 \\
8\end{array}$ & $\begin{array}{r}83.7 \\
15.5 \\
0.8\end{array}$ & . & & $\begin{array}{r}6706 \\
1280 \\
71\end{array}$ & $\begin{array}{r}83.2 \\
15.9 \\
0.9\end{array}$ & $\dot{r}$ & \\
\hline \multicolumn{9}{|l|}{ Cigarette smoking } \\
\hline $\begin{array}{l}\text { Never smoker } \\
\text { Ever smoker } \\
\text { Current smoker } \\
\text { Former smoker } \\
\text { Unknown }\end{array}$ & $\begin{array}{r}151 \\
909 \\
314 \\
564 \\
2\end{array}$ & $\begin{array}{r}14.2 \\
85.6 \\
29.6 \\
53.1 \\
0.2\end{array}$ & $\dot{.}$ & & $\begin{array}{r}1767 \\
6268 \\
1894 \\
4118 \\
22\end{array}$ & $\begin{array}{r}21.9 \\
77.8 \\
23.5 \\
51.1 \\
0.3\end{array}$ & $\begin{array}{l}\dot{ } \\
\dot{.} \\
\dot{ } \\
\dot{ }\end{array}$ & 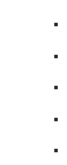 \\
\hline \multicolumn{9}{|l|}{ Ever smokers only } \\
\hline $\begin{array}{l}\text { Cigarettes per day } \\
\text { Years smoked } \\
\text { Pack-years }\end{array}$ & : & & $\begin{array}{l}21.3 \\
36.5 \\
33.6\end{array}$ & $\begin{array}{l}12.7 \\
14.9 \\
29.0\end{array}$ & 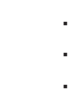 & & $\begin{array}{l}20.9 \\
33.5 \\
27.9\end{array}$ & $\begin{array}{l}12.5 \\
15.0 \\
28.3\end{array}$ \\
\hline \multicolumn{9}{|l|}{ Former smokers only } \\
\hline Years quit & . & & 16.8 & 11.9 & 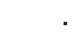 & & 18.3 & 12.6 \\
\hline \multicolumn{9}{|l|}{ Alcohol consumption } \\
\hline $\begin{array}{l}\text { Never } \\
\text { Ever } \\
\text { Unknown }\end{array}$ & $\begin{array}{l}113 \\
811 \\
138\end{array}$ & $\begin{array}{l}10.6 \\
76.4 \\
13.0\end{array}$ & : & . & $\begin{array}{r}842 \\
5882 \\
1333\end{array}$ & $\begin{array}{l}10.5 \\
73.0 \\
16.5\end{array}$ & . & \\
\hline \multicolumn{9}{|l|}{ Questionnaire respondent } \\
\hline $\begin{array}{l}\text { Patient } \\
\text { Proxy } \\
\text { Unknown }\end{array}$ & $\begin{array}{r}888 \\
150 \\
24\end{array}$ & $\begin{array}{r}83.6 \\
14.1 \\
2.3\end{array}$ & : & & $\begin{array}{r}6357 \\
1490 \\
210\end{array}$ & $\begin{array}{r}78.9 \\
18.5 \\
2.6\end{array}$ & . & \\
\hline
\end{tabular}

a Work years after 5 years prior to the diagnosis have been excluded. A work year is defined as 1 year in a full-time job or 2 years in a part-time job.

${ }^{b}$ Data missing due to the alcohol consumption question being added to the questionnaire during the first year of the study. answered the questionnaire themselves, and to have smoked (table 2). These potentially important confounding variables were adjusted for in all of the analyses.

The study participants reported an average of 4.6 jobs and 36 work years. Distributions of the types of occupations and industries have been previously reported (9). All Canadian occupations and industries could be translated into US equivalents, except for jobs in the armed forces $(4.6 \%)$, which were assumed to be unexposed to all chemical agents. Each reported Canadian job translated into an average of 3.5 US industry-occupation combinations. Fifty-two percent of the jobs in this study were not listed in the NOES-JEM. These include $25 \%$ of the jobs in industries that were excluded in the NOES survey $(14,15)$ either because they had low potential for any exposure (government $6 \%$, education $2 \%$, and finance, insurance and real estate $4 \%$ ) or because their exposures were thought to be too heterogeneous (agriculture 8\%, mining 4\%, and fishing and trapping $1 \%)$. The remaining jobs were in surveyed industries but were not listed in the NOES JEM, possibly due to low or no exposure, including retail and wholesalers $(10 \%)$, business and others services (6\%), and transportation (3\%). Overall, $74 \%$ of the study participants had some occupational exposure.

\section{Occupational chemical agents}

The ever exposure results for 5661 chemical agents with at least three exposed cases plus the additional dose-response results for 3419 chemical agents with at least

Table 2. Odds ratios (OR) for potentially important ${ }^{\text {a }}$ confounding variables in a bladder cancer study, British Columbia, Canada, 1983-1990. (95\% Cl = 95\% confidence interval)

\begin{tabular}{lrcc}
\hline Confounding variable & $\begin{array}{c}\text { Cases } \\
(\mathrm{N})\end{array}$ & OR & $95 \% \mathrm{Cl}$ \\
& & & \\
\hline Respondent to questionnaire & & & \\
$\quad$ Patient & 888 & 1.00 &.. \\
Proxy & 150 & 0.65 & $0.53-0.78$ \\
$\quad$ Unknown & 24 & 0.92 & $0.59-1.42$ \\
Ethnic origin & & & \\
Caucasian & 1027 & 1.00 &.. \\
$\quad$ Non-Caucasian & 31 & 0.71 & $0.48-1.05$ \\
Unknown & 4 & 0.63 & $0.22-1.79$ \\
Alcohol consumption status & & & \\
$\quad$ Never drinker & 113 & 1.00 &.. \\
Ever drinker & 811 & 0.88 & $0.70-1.11$ \\
$\quad$ Unknown & 138 & 1.20 & $0.87-1.67$ \\
Cigarette smoking duration & & & \\
0 years & 151 & 1.00 &.. \\
1-29 years & 262 & 1.41 & $1.13-1.75$ \\
30-44 years & 338 & 1.93 & $1.56-2.40$ \\
$\geq 45$ years & 300 & 2.36 & $1.89-2.95$ \\
Unknown & 11 & 1.16 & $0.60-2.23$ \\
\hline
\end{tabular}

a P-value $<0.20$. 
nine exposed cases are listed in appendix 1, which can be found on the homepage of the Scandinavian Journal of Work, Environment \& Health.

Chemical agents with three or more exposed cases. $\mathrm{Al}-$ though 283 chemical agents were expected to be significant by chance alone, a significantly increased OR for ever exposure was detected for 635 chemical agents, of which 163 had OR values above two. After adjustment for multiple comparisons, 7 chemical agents remained significant (table 3).

Chemical agents with nine or more exposed cases. Although 171 chemical agents were expected to exhibit a significant $\mathrm{OR}$ value for ever exposure by chance alone, a total of 502 chemical agents had a significantly increased OR value. Although 171 chemical agents were also independently expected to have a significant linear trend test result by chance alone, a significantly increasing linear trend was demonstrated for 341 chemical agents. A total of 298 chemical agents showed both results as significant. After adjustment for multiple comparisons, 24 and 22 chemical agents continued to demonstrate either a significant OR for ever exposure or a significant linear trend, respectively, with 17 chemical agents showing both. Altogether 29 different chemical

Table 3. Conditional logistic regression-adjusted ${ }^{\text {a }}$ risk estimates for ever exposure to seven chemical agents with significant associations after adjustment for multiple testing in a bladder cancer study, British Columbia, Canada, 1983-1990. [OR = odds ratio; $95 \% \mathrm{Cl}=95 \%$ confidence interval; $\mathrm{M}=$ mixed 0,0 -bis(sec-bu and 1,3-dimethylbutyl) esters; $Z S$ = zinc salt; $A E=1-(2-((2-((2-((2-$ aminoethyl)amino)ethyl)amino)ethyl)amino)ethyl)-, monopolyisobutenyl derivatives; $\mathrm{RP}=$ reaction products; $\mathrm{ClH}=$ chlorinated isobutylene homopolymer; $2 \mathrm{~B}=2$-(2-(2-butoxyethoxy)ethoxy)-; $\mathrm{S}=$ sulfurized dodecylphenol calcium salt, sulfurized; $\mathrm{C}=$ carbonates, calcium salts, overbased]

\begin{tabular}{|c|c|c|c|}
\hline \multirow[t]{2}{*}{ NOES chemical agent ${ }^{\mathrm{b}}$} & \multirow{2}{*}{$\begin{array}{l}\text { Cases } \\
\text { (N) }\end{array}$} & \multicolumn{2}{|c|}{ Ever exposure $^{c}$} \\
\hline & & $\mathrm{OR}$ & $95 \% \mathrm{Cl}$ \\
\hline \multicolumn{4}{|l|}{ Petroleum product or additive } \\
\hline Phosphorodithioic acid, M ZS (X2306) & 335 & 1.38 & $1.20-1.60$ \\
\hline \multicolumn{4}{|l|}{ Lubricating oil or grease } \\
\hline 2,5-Pyrrolidinedione, AE (X2303) & 361 & 1.39 & $1.21-1.60$ \\
\hline \multicolumn{4}{|l|}{ Solvent or paint } \\
\hline 1, 2-Ethanediamine, RP with CIH (X2689) & 25 & 2.89 & $1.79-4.67$ \\
\hline Ethanol, 2B (M0984) & 176 & 1.48 & $1.23-1.77$ \\
\hline \multicolumn{4}{|l|}{ Soap or detergent } \\
\hline Alkenes, c15-18 alpha-, RP with S (X2307) & 301 & 1.38 & $1.19-1.60$ \\
\hline Phenol, dodecyl-, sulfurized, C (X2298) & 390 & 1.34 & $1.16-1.53$ \\
\hline \multicolumn{4}{|l|}{ Other } \\
\hline Natural gas, liquefied (Y1006) & 25 & 3.11 & $1.92-5.04$ \\
\hline
\end{tabular}

agents were identified as potentially exhibiting bladdercancer carcinogenic properties. The results are shown in table 4.

Among the identified chemical agents, several are petroleum products or additives. Others include chemical agents used in the formulation of lubricant oils, engine oils and grease; those used in solvents or paints; and soaps and detergents (tables 3 and 4).

\section{Principal component analysis}

Performing a principal component analysis on the identified 29 chemical agents resulted in five principal components (table 5). The largest principal component accounted for $26 \%$ of the total variance, and the five principal components combined accounted for more than $75 \%$ of the total variance. The chemical agents tended to be grouped according to the jobs in which their exposures mainly occurred. Exposures comprising the first and second principal components were predominantly due to employment in the logging and gasoline service station industries, respectively. The third principal component mainly consisted of exposures from the construction and automotive repair shop industries. A considerable proportion of the exposures in the fourth principal component were due to employment as a truck driver. Members of the fourth and fifth principal components also had exposures in the gasoline service station industry.

\section{Chemical agents identified by the International Agency for Research on Cancer}

Table 6 presents the ever exposure and dose-response results for chemical agents identified by $\operatorname{IARC}(6,7)$. Among the seven chemical agents with available data for evaluation, statistically significant or at least a 50\% increased risk was observed for four chemical agents (mineral oils, benz(a)anthracene, 4-chloro-ortho-toluidine, and diesel engine exhaust) which also exhibited a significant dose-response relationship $(\mathrm{P}=0.01)$. Furthermore, a borderline significant increased risk was found for coal-tar pitches (OR 1.42, 95\% CI 0.99-2.04). A nonsignificant $36 \%$ increased risk was found for direct black 38 , a benzidine-based dye. No excess risk was observed for ortho-toluidine.

\section{Discussion}

Many characteristics of this study strengthen our findings. Both the cases and controls were selected from population-based cancer incidence patients, and all of the cancers were histologically confirmed. The large 
Table 4. Conditional logistic regression-adjusted ${ }^{\text {a }}$ risk estimates for occupational exposure to 29 selected chemical agents with significant associations in a bladder cancer study, British Columbia, Canada, 1983-1990. [OR = odds ratio; $95 \% \mathrm{Cl}=95 \%$ confidence interval; RP = reaction products; $\mathrm{ClH}=$ chlorinated isobutylene homopolymer; $\mathrm{AE}=1-(2-((2-((2-((2$-aminoethyl)amino)ethyl)amino)ethyl)amino)ethyl)-, monopolyisobutenyl derivatives; PW1 = polymer with 1,3-butadiene and ethenylbenzene; $2 \mathrm{ME}=2$-me-, c12 ester, poly w/ c16 2me2propenoate, iso-c10 2me2propenoate, me 2me2propenoate, c18 2me2propenoate, c14 2me2propenoate; $\mathrm{S}$ = sulfurized dodecylphenol calcium salt, sulfurized; $\mathrm{NEC}=$ not elsewhere classified; $2 \mathrm{~B}=2-(2-(2$-butoxyethoxy)ethoxy)-; $\mathrm{OA}=0$ oxide adduct; $\mathrm{C}=$ carbonates, calcium salts, overbased; CSO = calcium salts, overbased; $M=$ mixed 0, 0-bis(sec-bu and 1,3-dimethylbutyl) esters; ZS = zinc salt; 00B = 0, 0-bis(2ethylhexyl) ester; 02E = 0-(2-ethylhexyl) 0-isobutyl ester; $\mathrm{POC}$ = products of combustion; $\mathrm{U}=$ unleaded; RHPD = refined heavy paraffinic distillate; MS = magnesium salts]

\begin{tabular}{|c|c|c|c|c|c|c|c|c|c|c|}
\hline \multirow[t]{3}{*}{ NOES chemical agent ${ }^{b}$} & \multicolumn{3}{|c|}{ Ever exposure ${ }^{c}$} & \multicolumn{6}{|c|}{ Dose-response ${ }^{c}$} & \multirow{3}{*}{$\begin{array}{c}\text { P-value } \\
\text { (linear } \\
\text { trend) }\end{array}$} \\
\hline & \multirow[t]{2}{*}{ Cases } & \multirow[t]{2}{*}{$\mathrm{OR}$} & \multirow[t]{2}{*}{$95 \% \mathrm{Cl}$} & \multicolumn{2}{|r|}{ Low } & \multicolumn{2}{|c|}{ Medium } & \multicolumn{2}{|c|}{ High } & \\
\hline & & & & $\mathrm{OR}$ & $95 \% \mathrm{Cl}$ & $\mathrm{OR}$ & $95 \% \mathrm{Cl}$ & $\mathrm{OR}$ & $95 \% \mathrm{Cl}$ & \\
\hline \multicolumn{11}{|l|}{ Petroleum product or additive } \\
\hline Asphalt (90320) & 499 & 1.29 & $1.13-1.47$ & 1.40 & $1.16-1.69$ & 1.13 & $0.93-1.37$ & 1.34 & $1.11-1.62$ & 0.0018 \\
\hline Ether, tert - butyl methyl (X4267) & 32 & 1.96 & $1.31-2.93$ & 1.19 & $0.56-2.56$ & 2.40 & $1.23-4.70$ & 2.56 & $1.30-5.02$ & 0.0003 \\
\hline Phosphorodithioic acid, M ZS (X2306) & 335 & 1.38 & $1.20-1.60$ & 1.40 & $1.13-1.75$ & 1.41 & $1.13-1.76$ & 1.34 & $1.07-1.68$ & 0.0001 \\
\hline Phosphorodithioic acid, OOB ZS (X2295) & 450 & 1.30 & $1.14-1.49$ & 1.25 & $1.02-1.52$ & 1.35 & $1.11-1.64$ & 1.31 & $1.08-1.60$ & 0.0003 \\
\hline Phosphorodithioic acid, 02E ZS (X1075) & 161 & 1.42 & $1.17-1.71$ & 1.22 & $0.88-1.69$ & 1.59 & $1.18-2.13$ & 1.44 & $1.06-1.95$ & 0.0003 \\
\hline POC: gasoline (leaded) (60713) & 617 & 1.26 & $1.10-1.44$ & 1.15 & $0.95-1.38$ & 1.27 & $1.06-1.52$ & 1.36 & $1.14-1.62$ & 0.0002 \\
\hline POC: jet fuel \& gasoline, U (X5263) & 557 & 1.28 & $1.12-1.46$ & 1.24 & $1.02-1.49$ & 1.24 & $1.03-1.49$ & 1.37 & $1.14-1.65$ & 0.0003 \\
\hline Sulfonic acids, petroleum, C (X2293) & 375 & 1.30 & $1.13-1.49$ & 1.26 & $1.02-1.56$ & 1.34 & $1.09-1.65$ & 1.30 & $1.05-1.61$ & 0.0006 \\
\hline Sulfonic acids, petroleum, MS (X2308) & 208 & 1.40 & $1.18-1.66$ & 1.25 & $0.94-1.67$ & 1.34 & $1.02-1.77$ & 1.61 & $1.24-2.09$ & $<0.0001$ \\
\hline \multicolumn{11}{|l|}{ Lubricating oil or grease } \\
\hline 2-Butenedioic acid (e)-, PW1 (X1401) & 35 & 2.18 & $1.47-3.22$ & 1.62 & $0.78-3.39$ & 2.68 & $1.45-4.96$ & 2.25 & $1.12-4.49$ & 0.0001 \\
\hline Oil, hydraulic (92500) & 51 & 1.74 & $1.26-2.39$ & 0.80 & $0.38-1.69$ & 2.30 & $1.41-3.75$ & 2.15 & $1.28-3.60$ & $<0.0001$ \\
\hline 1-Propene, 2-methyl-, sulfurized (X9078) & 397 & 1.27 & $1.11-1.46$ & 1.11 & $0.89-1.38$ & 1.30 & $1.06-1.59$ & 1.39 & $1.14-1.70$ & 0.0001 \\
\hline 2-Propenoic acid, 2ME (X1894) & 48 & 1.86 & $1.34-2.60$ & 1.63 & $0.88-3.02$ & 1.73 & $0.97-3.10$ & 2.20 & $1.30-3.73$ & 0.0002 \\
\hline 2,5-Pyrrolidinedione, AE RP (X2305) & 206 & 1.38 & $1.17-1.64$ & 1.22 & $0.92-1.63$ & 1.32 & $0.99-1.74$ & 1.62 & $1.25-2.10$ & $<0.0001$ \\
\hline 2,5-Pyrrolidinedione, AE (X2303) & 361 & 1.39 & $1.21-1.60$ & 1.42 & $1.15-1.75$ & 1.42 & $1.15-1.76$ & 1.33 & $1.07-1.66$ & $<0.0001$ \\
\hline \multicolumn{11}{|l|}{ Solvent or paint } \\
\hline 1, 2-Ethanediamine, RP with CIH (X2689) & 25 & 2.89 & $1.79-4.67$ & 1.93 & $0.77-4.85$ & 2.95 & $1.33-6.55$ & 4.00 & $1.81-8.82$ & $<0.0001$ \\
\hline Cyclohexylamine, n-ethyl- (M1150) & 28 & 2.29 & $1.48-3.54$ & 2.31 & $1.15-4.64$ & 3.59 & $1.83-7.06$ & 1.03 & $0.36-2.98$ & 0.0035 \\
\hline Ethanol, 2B (M0984) & 176 & 1.48 & $1.23-1.77$ & 1.57 & $1.18-2.10$ & 1.34 & $0.99-1.82$ & 1.52 & $1.13-2.03$ & 0.0002 \\
\hline Heptane $(36060)$ & 457 & 1.30 & $1.14-1.49$ & 1.35 & $1.11-1.64$ & 1.22 & $1.00-1.49$ & 1.33 & $1.10-1.62$ & 0.0008 \\
\hline Hexane (36955) & 477 & 1.30 & $1.14-1.48$ & 1.44 & $1.19-1.74$ & 1.25 & $1.03-1.52$ & 1.21 & $0.99-1.47$ & 0.0071 \\
\hline Solvent RHPD (petroleum) (T1475) & 535 & 1.24 & $1.08-1.41$ & 1.18 & $0.97-1.42$ & 1.09 & $0.90-1.32$ & 1.45 & $1.21-1.73$ & 0.0002 \\
\hline \multicolumn{11}{|l|}{ Soap or detergent } \\
\hline Alkenes, c15-18 alpha-, RP with S (X2307) & 301 & 1.38 & $1.19-1.60$ & 1.40 & $1.12-1.75$ & 1.39 & $1.11-1.74$ & 1.36 & $1.07-1.74$ & 0.0001 \\
\hline Nonylphenol ethylene OA (T1909) & 80 & 1.63 & $1.26-2.11$ & 1.17 & $0.72-1.91$ & 1.76 & $1.16-2.68$ & 2.01 & $1.32-3.06$ & $<0.0001$ \\
\hline Nonylphenoxyethanol (83048) & 27 & 2.49 & $1.59-3.90$ & 2.08 & $0.93-4.65$ & 2.61 & $1.20-5.69$ & 2.81 & $1.33-5.94$ & 0.0001 \\
\hline Phenol, dodecyl-, sulfurized, C (X2298) & 390 & 1.34 & $1.16-1.53$ & 1.33 & $1.08-1.63$ & 1.41 & $1.15-1.72$ & 1.27 & $1.03-1.57$ & 0.0004 \\
\hline \multicolumn{11}{|l|}{ Other } \\
\hline Clay, not elsewhere classified (90590) & 375 & 1.29 & $1.13-1.48$ & 1.33 & $1.09-1.64$ & 1.27 & $1.02-1.57$ & 1.28 & $1.03-1.57$ & 0.0020 \\
\hline Natural gas, liquefied (Y1006) & 25 & 3.11 & $1.92-5.04$ & 2.70 & $1.40-5.20$ & 2.46 & $0.48-12.56$ & 4.12 & $1.85-9.16$ & $<0.0001$ \\
\hline Sn, tin - MF unknown (73075) & 420 & 1.30 & $1.13-1.48$ & 1.22 & $0.99-1.49$ & 1.30 & $1.06-1.59$ & 1.38 & $1.13-1.68$ & 0.0002 \\
\hline OFW steel (S2599) & 221 & 1.37 & $1.16-1.61$ & 1.45 & $1.12-1.87$ & 1.37 & $1.05-1.79$ & 1.28 & $0.97-1.67$ & 0.0022 \\
\hline
\end{tabular}

${ }^{a}$ Matched for age and year of diagnosis; additionally adjusted for ethnicity, years of smoking, alcohol consumption, and questionnaire respondent.

${ }^{b}$ Code of the National Occupational Exposure Survey (NOES) in parentheses.

${ }^{\circ}$ See the text for an explanation of ever and dose-response exposure levels. The zero cumulative exposure group was always used as the reference category.

number of cases and controls enabled us to evaluate risk on the basis of exposure to a specific chemical agent rather than according to less precise employment categories. Applying a generic job-exposure matrix enabled us to study a range of specific chemical agents, usually prohibitive for studies utilizing expert assessments. The exposure estimates were also more reproducible and objective than those from the expert assessments (22). Over $80 \%$ of the patients completed the questionnaire themselves, and the questionnaire was found to be valid and reliable (10). Differential recall bias was minimized because cancer controls were used. Complete job histories were provided, enabling more precise measures of cumulative exposure through the incorporation of both estimated probability and the duration of exposure within all jobs. Besides adjusting for confounding factors, we controlled for multiple comparisons in the analysis to reduce the chance of false positives.

A limitation of this study, common for studies utilizing a job-exposure matrix, was the potential for 
Table 5. Principal component analysis loadings a for occupational exposure to 29 selected chemical agents, British Columbia, Canada, 1983-1990. [NOES = National Occupational Exposure Survey; $\mathrm{RP}=$ reaction products; $\mathrm{CIH}=$ chlorinated isobutylene homopolymer; $\mathrm{AE}$ = 1-(2-((2-((2-((2-aminoethyl)amino)ethyl)amino)ethyl)amino)ethyl)-, monopolyisobutenyl derivatives; PW1 = polymer with 1,3-butadiene and ethenylbenzene; $2 \mathrm{ME}=2-\mathrm{me}-, \mathrm{c} 12$ ester, poly w/ c16 2me2propenoate, iso-c10 2me2propenoate, me $2 \mathrm{me} 2$ propenoate, c18 2me2propenoate, c14 2me2propenoate; $S$ = sulfurized dodecylphenol calcium salt, sulfurized; 2B = 2-(2-(2-butoxyethoxy)ethoxy)-; 0A = oxide adduct; $\mathrm{C}$ = carbonates, calcium salts, overbased; $\mathrm{CSO}=$ calcium salts, overbased; $\mathrm{M}=$ mixed 0, 0-bis(sec-bu and 1,3-dimethylbutyl) esters; ZS = zinc salt; OOB = 0, 0-bis(2-ethylhexyl) ester; 02E = 0-(2-ethylhexyl) 0-isobutyl ester; POC = products of combustion; $U$ = unleaded; RHPD = refined heavy paraffinic distillate; MS = magnesium salts]

\begin{tabular}{|c|c|c|c|c|c|}
\hline \multirow[t]{2}{*}{ NOES chemical agent ${ }^{\mathrm{b}}$} & \multicolumn{5}{|c|}{ Principal component } \\
\hline & 1 & 2 & 3 & 4 & 5 \\
\hline Alkenes, c15-18 alpha-, RP with S (X2307) & $93^{c}$ & 8 & 1 & 15 & 0 \\
\hline Asphalt (90320) & $58^{c}$ & -4 & $57^{c}$ & 9 & 8 \\
\hline 2-Butenedioic acid (e)-, PW1 (X1401) & 9 & $88^{c}$ & 6 & 6 & 5 \\
\hline Clay, not elsewhere classified (90590) & 17 & -7 & $73^{c}$ & 7 & 22 \\
\hline Cyclohexylamine, n-ethyl- (M1150) & 10 & $84^{c}$ & 5 & 4 & 2 \\
\hline 1, 2-Ethanediamine, RP with CIH (X2689) & 5 & $89 c$ & 2 & 8 & 14 \\
\hline Ethanol, 2B (M0984) & 1 & $41^{c}$ & $67^{c}$ & 18 & -24 \\
\hline Ether, tert - butyl methyl (X4267) & 8 & $90^{c}$ & 7 & 5 & 2 \\
\hline Heptane (36060) & 22 & 5 & $79^{c}$ & 0 & 15 \\
\hline Hexane (36955) & 19 & 2 & $78^{c}$ & 1 & 21 \\
\hline Natural gas, liquefied (Y1006) & 5 & $90^{c}$ & 2 & 8 & 14 \\
\hline Nonylphenol ethylene OA (T1909) & 14 & $51^{c}$ & 16 & 15 & $56^{c}$ \\
\hline Nonylphenoxyethanol (83048) & 8 & $95^{c}$ & 3 & 6 & 7 \\
\hline OFW steel (S2599) & 6 & 19 & $76^{c}$ & 15 & -20 \\
\hline Oil, hydraulic (92500) & 6 & $55^{c}$ & 10 & 12 & $64^{c}$ \\
\hline Phenol, dodecyl-, sulfurized, C (X2298) & $83^{c}$ & 10 & 25 & 17 & -3 \\
\hline Phosphorodithioic acid, M ZS (X2306) & $92^{c}$ & 9 & 2 & 23 & 8 \\
\hline Phosphorodithioic acid, 02E ZS (X2295) & 22 & 28 & 35 & $65^{c}$ & -21 \\
\hline Phosphorodithioic acid, OOB ZS (X1075) & $87^{c}$ & 4 & 15 & 13 & 17 \\
\hline POC - gasoline (leaded) (X9078) & $61^{c}$ & 10 & $50^{c}$ & 0 & -16 \\
\hline POC - jet fuel \& gasoline, U (X1894) & $64^{c}$ & 13 & $51^{c}$ & 1 & -20 \\
\hline 1-Propene, 2-methyl-, sulfurized (X2305) & $72^{c}$ & 9 & $41^{\mathrm{c}}$ & -19 & 4 \\
\hline 2-Propenoic acid, 2ME (X2303) & 3 & $49^{c}$ & 13 & 14 & $51^{c}$ \\
\hline 2,5-Pyrrolidinedione, AE (60713) & $89^{c}$ & 11 & 14 & 28 & 0 \\
\hline 2,5-Pyrrolidinedione, AE RP (X5263) & $40^{c}$ & 12 & 4 & $83^{c}$ & 22 \\
\hline Sn, tin - MF unknown (73075) & 20 & -3 & $74^{c}$ & 2 & 5 \\
\hline Solvent RHPD (petroleum) (T1475) & $63^{c}$ & 6 & $40^{c}$ & 17 & 4 \\
\hline Sulfonic acids, petroleum, C (X2293) & $91^{c}$ & 6 & 8 & 19 & 12 \\
\hline Sulfonic acids, petroleum, MS (X2308) & $41^{c}$ & 12 & 5 & $83^{c}$ & 23 \\
\hline Percentage of variance explained & 25.5 & 20.6 & 16.7 & 7.8 & 5.2 \\
\hline Cumulative percentage & 25.5 & 46.2 & 62.8 & 70.6 & 75.9 \\
\hline
\end{tabular}

a The component loadings have been multiplied by 100 and rounded to the nearest integer.

${ }^{b}$ Code of the National Occupational Exposure Survey (NOES) in parentheses

${ }^{c}$ The component loadings were greater than plus or minus 0.4 .

misclassifying exposure. It is, however, more likely to be nondifferential and may underestimate the true association; thus negative findings should be regarded as inconclusive (23). Assuming zero exposure for industries not studied by NOES may not be realistic since excess bladder cancer has been suspected in some of these industries, such as agricultural production and mining (1, $5,24)$. However, these industries did not exhibit excess risk in this study population (9). Assuming no exposure for jobs in the armed forces may also be unrealistic for some exposures (eg, combustion products). However, no excess was observed for this group in an earlier analysis (9). Furthermore, the estimated OR values from the current study changed only slightly $(<10 \%)$, with no changes in significant results, when patients ever in the armed forces were excluded. We averaged the exposure probabilities over all of the translated US industry-occupation combinations, as we did not have information on which translations were more likely. We also assumed the exposure was zero when a translated US industry-occupation combination was not in the job-exposure matrix, as we were unable to distinguish between it being invalid in the United States or having been observed to be unexposed. Thus cumulative exposures were subject to some measurement error from averaging over exposures for invalid or inappropriate 
Table 6. Conditional logistic regression-adjusted a risk estimates for occupational exposure to definite and probable bladder carcinogens in a study in British Columbia, Canada, 1983-1990. [NOES-JEM = job-exposure matrix based on the National Occupational Exposure Survey, $\mathrm{OR}=$ odds ratio, $95 \% \mathrm{Cl}=95 \%$ confidence interval]

\begin{tabular}{|c|c|c|c|c|c|c|c|c|c|c|}
\hline \multirow[t]{3}{*}{ Chemical agent ${ }^{b, c}$} & \multicolumn{3}{|c|}{ Ever exposure ${ }^{d}$} & \multicolumn{6}{|c|}{ Dose-response ${ }^{d}$} & \multirow{3}{*}{$\begin{array}{l}\text { P-value } \\
\text { (linear } \\
\text { trend) }\end{array}$} \\
\hline & \multirow[t]{2}{*}{ Cases } & \multirow[t]{2}{*}{$\mathrm{OR}$} & \multirow[t]{2}{*}{$95 \% \mathrm{Cl}$} & \multicolumn{2}{|c|}{ Low } & \multicolumn{2}{|c|}{ Medium } & \multicolumn{2}{|c|}{ High } & \\
\hline & & & & $\mathrm{OR}$ & $95 \% \mathrm{Cl}$ & $\mathrm{OR}$ & $95 \% \mathrm{Cl}$ & $\mathrm{OR}$ & $95 \% \mathrm{Cl}$ & \\
\hline Coal-tar pitches (90620) & 39 & 1.42 & $0.99-2.04$ & 1.50 & $0.82-2.76$ & 1.27 & $0.66-2.44$ & 1.49 & $0.82-2.68$ & 0.08 \\
\hline Mineral oils (M0603) & 467 & 1.16 & $1.01-1.32$ & 1.19 & $0.98-1.44$ & 1.25 & $1.04-1.51$ & 1.03 & $0.84-1.26$ & 0.18 \\
\hline \multicolumn{11}{|l|}{ Polyaromatic hydrocarbons } \\
\hline Benz(a)anthracene (B0045) & 13 & 1.92 & $1.02-3.61$ & 2.65 & $1.03-6.82$ & 1.38 & $0.39-4.90$ & 1.69 & $0.55-5.23$ & 0.12 \\
\hline Diesel engine exhaust (80017) & 605 & 1.18 & $1.04-1.35$ & 1.14 & $0.95-1.37$ & 1.17 & $0.97-1.40$ & 1.25 & $1.04-1.49$ & 0.01 \\
\hline \multicolumn{11}{|l|}{ Benzidine-based dyes } \\
\hline Direct black 38 (M1410) & 12 & 1.36 & $0.72-2.56$ & 1.55 & $0.52-4.64$ & 1.14 & $0.39-3.34$ & 1.46 & $0.48-4.42$ & 0.39 \\
\hline 4-Chloro-ortho-toluidine (M4543) & 7 & 1.76 & $0.76-4.08$ & & & & & & & \\
\hline ortho-Toluidine (73470) & 39 & 1.01 & $0.71-1.44$ & 1.49 & $0.89-2.52$ & 0.46 & $0.20-1.07$ & 1.10 & $0.62-1.94$ & 0.79 \\
\hline
\end{tabular}

a Matched for age and year of diagnosis; additionally adjusted for ethnicity, years of smoking, alcohol consumption, and questionnaire respondent. The results are presented for chemical agents with at least three exposed cases. Data were not available for chemical agents that were not included in the NOES-JEM. When multiple NOES-JEM equivalents were found for a bladder carcinogen, the results for the one with the greatest number of exposed cases were given.

${ }^{b}$ Code of the National Occupational Exposure Survey (NOES) in parentheses.

c No NOES-JEM chemical agent equivalent could be found for 4-aminobiphenyl and dibenz(a,h)anthracene. Fewer than three exposed cases were found for benzidine, 2-naphthylamine, benzo[a]pyrene, 4,4'-methylene bis(2-chloroaniline), and benzidine-based dyes direct blue 6 and direct brown 95.

d See the text for an explanation of the ever and dose-response exposure levels. The zero cumulative exposure group was always used as the reference category.

e The P-value in italics represents a decreasing linear trend.

US translations, but the degree of error should not have differed between the cases and controls.

The compatibility of exposure levels between the US industries and Canadian or British Columbia equivalences is a concern. However a comparison of the jobexposure matrix specifically developed for the lead and zinc industry in British Columbia and that from the NOES-JEM showed good concordance overall (25).

We were also unable to account for variations in the concentration or frequency of exposure, variation within jobs, or changes across time. Although the NOES-JEM may not be very specific in comparison with expert assessments, self-reports, and other job-exposure matrices (26), our study found consistent results for definite or probable bladder carcinogens. There is also the potential for selection bias due to the use of cancer controls, a procedure which biases estimates towards unity (27). Lung cancer controls were excluded and a range of other cancer controls was used to limit this bias (19). Nonresponse bias has been examined earlier and found to be minimal (10). By various means, minimal information, including years of schooling, ever smoking, and usual occupation, was obtained for 227 nonrespondents. Comparisons with the respondents showed no significant differences, except for managerial occupations. Questionnaire reliability and recall bias were also examined, and excellent concordance was observed with interclass correlations in the range of 0.95 (10).

This case-control study showed a significant positive association between the risk of developing bladder cancer and exposure to several specific chemical agents, after adjustment for confounding factors and multiple testing (tables 3 and 4). The exposures were based on the NOES-JEM with specific chemical agents, and relatively few of them were studied in association with cancer. Most of the identified chemical agents can, however, be broadly grouped into subcategories as petroleum products or additives, lubricating oils and grease, paints and solvents, and soaps and detergents.

For petroleum products and additives, we observed a significantly elevated risk for asphalt and gasoline combustion, in addition to other specific chemicals that have, to our knowledge, not been reported previously in the literature. Asphalt (bitumen) has remained unclassifiable by IARC; however, it is on its high-priority list for reconsideration (6). A few other studies have reported a positive association $(28,29)$. Gasoline and diesel engine exhaust are currently classified by IARC as probably and possibly carcinogenic, respectively (30). Several other studies have indicated an increased risk for exposure to petroleum and combustion products, suggesting an association with PAH (7, 31-34). An earlier analysis of this case-control study on the basis of occupations and industries also suggested a link with PAH exposure (9) through employment in asphalt paving, truck driving, mechanic work, gasoline service station attending, printing, painting, and the metal and aluminum industries. IARC has classified some PAH as probably carcinogenic and many of the occupational circumstances with high PAH exposure as definitely or probably carcinogenic (35). 
We found a significantly increased risk for several specific chemicals used in the formulation of engine oils, lubricating oils, or grease. Although these chemicals have generally never been studied individually in relation to cancer, a recent study from the four western Canadian provinces has found significant excess risk for exposure to lubricating, cutting, and mineral oils (36). Several past studies have also observed strong evidence for such an association $(7,37-39)$. It is interesting to note that a recent biological study has found that exposure to PAH or other compounds from skin contact with engine oils may cause oxidative DNA (deoxyribonucleic acid) damage (40), suggesting that a dermal absorption of carcinogens can lead to cancer development.

Several specific solvents, some used, for example, as components of polymer paints, have been observed to have a significant association with the risk of developing bladder cancer in this study. Past case-control and cohort studies have suggested an association with exposure to solvents and paints $(5,41-43)$. A recent study in the Netherlands found a nonsignificant excess for those exposed to paint components (33). However, only general components were used in these studies; therefore, information on their association with specific chemical agents was not available.

In this study, a few specific surfactants used in soaps and detergents were significantly associated with bladder cancer risk. These specific chemical agents have not been reported individually in the literature. However, an excess risk for exposure to soaps and detergents was observed in a mortality study (44).

Significant excess risk was observed for clay in this study. Exposure to clay dust was found to be associated with bladder cancer in a Montreal study (8). The fact that clay dust is a common exposure within the clay-, glass-, and stone-processing industries may explain why an excess risk for exposure to clay was found in this study.

This dataset was originally analyzed with the use of job title and industry. Within each job or industry, workers may possibly be exposed to several hundred chemical agents. Looking at exposure to individual chemical agents, as in this study, led us to a smaller subset of potentially important chemicals. However workers may be still exposed to several chemicals within this smaller subset concurrently since several chemicals may be present together in a given job. The principal component analysis was thus used to identify subgroups of chemical agents (commonly called components) for which workers tended to be exposed together within this subset. For each subgroup, the results provided chemical agents that may individually or collectively contribute to an increased risk of bladder cancer. Due to high correlations, it is generally not possible to determine whether the chemical agents within each subgroup are individually or synergistically responsible for the observed association. However, the results could be used to suggest possible causes for increased risk on the basis of common characteristics of chemical agents within each subgroup.

The results from the principal components analysis suggest that the risk from the first component could be due to exposure to PAH (table 5). Many of the constituents are comprised of PAH or involve potential PAH exposure, such as asphalt and products of the combustion of gasoline (leaded and unleaded) and jet fuel. The remaining constituents of this component are ingredients for various engine, compressor, and lubricating oils, which could also have carcinogenic properties due to certain mineral and fuel oils being classified by IARC as definitely or possibly carcinogenic, respectively (6).

Exposures contributing to the second component were mainly due to employment in gasoline service stations. Excess bladder risk has been noted for this industry in this population and elsewhere $(1,9)$. Various engine emissions, diesel or traffic fumes, $\mathrm{PAH}$, lubricating oils, and greases could be responsible for the excess risk. Only service station attendants had possible exposure to liquefied natural gas, and, as such, were potentially exposed to natural gas combustion products, which were noted as potential carcinogens in a Montreal study (8). Ether, tert-butyl methyl is still unclassified by IARC (45) due to inadequate evidence with respect to human exposure; however, there is need for further research into this chemical agent as a potential carcinogen (46).

The construction and automotive repair industries contributed much of the exposures in the third component. Excess risk could be due to exposure to oils, solvents, or metal dusts. Clay and various metals have been suggested as bladder carcinogens $(8,9)$. A positive association of marginal significance between tin exposure and bladder cancer was also observed in a German case-control study (47).

There are no clear carcinogens in the remaining two components, but many of the chemical agents are again oils or products of petroleum. A significant proportion of the exposures in the fourth component were due to employment as a truck driver, which has been highlighted as at excess risk in many studies (47-49).

Results from our study are fairly consistent for definite or probable bladder carcinogens as classified by IARC (table 6). Of the specific PAH, we observed a large and significant excess risk for ever exposure to benz(a)anthracene and a borderline significant association for coal-tar pitch. Coal tar is a known carcinogen and a probable contributor of excess risk in the aluminum industry $(1,6,50)$. It should be noted that benzidine-based dyes and ortho-toluidine can occur in painting and printing, and they contain aromatic amines. They are both classified as probable carcinogens by IARC $(6,51)$. Benzidine-based dyes exhibited excess 
risk, although not at a statistically significant level, and ortho-toluidine did not exhibit any major excess risk in our study.

This study also replicated known associations between bladder cancer and Caucasian ethnicity and cigarette smoking (1). Smoking duration had a strong association with bladder cancer; yet it was thought to have a minimal confounding effect on the exposure results of the chemical agents. We examined the risk of exposure to the 29 selected chemical agents without adjusting for smoking duration, and all but two had a $<10 \%$ change in their OR values. The larger changes for the two chemical agents may be due to the smaller number of cases $(\mathrm{N}=25)$ and hence to a greater variation.

In conclusion, in our study, the use of a generic job-exposure matrix to estimate cumulative exposure corroborated important findings from the literature and documented bladder cancer risk for specific chemical agents not previously reported.

\section{Acknowledgments}

This study was funded by grants from the Workers' Compensation Board of British Columbia, the National Health Research and Development Program, Health Canada, and the Michael Foundation for Health Research.

The authors gratefully acknowledge Dr W Karl Sieber for providing the NOES-JEM, and the assistance of Raymond Fang, Donna Kan, and Barbara Lang.

\section{References}

1. Silverman DT, Hartge P, Morrison AS, Devesa SS. Epidemiology of bladder cancer. Hematol Oncol Clin North A. 1992;6:1-30.

2. Canadian Cancer Society/National Cancer Institute of Canada. Canadian cancer statistics 2007. Toronto (Canada): Canadian Cancer Society/National Cancer Institute of Canada; 2007.

3. Zeegers MP, Kellen E, Buntinx F, van den Brandt PA. The association between smoking, beverage consumption, diet and bladder cancer: a systematic literature review. World J Urol. 2004;21(6):392-401.

4. Vineis P, Simonato L. Proportion of lung and bladder cancers in males resulting from occupation: a systematic approach. Arch Environ Health. 1991;46:6-15.

5. Kogevinas M, 't Mannetje A, Cordier S, Ranft U, Gonzalez CA, Vineis $P$, et al. Occupation and bladder cancer among men in Western Europe. Cancer Causes Control. 2003;14:907-14.

6. International Agency for Research on Cancer (IARC). Overall evaluations of carcinogenicity: an updating of IARC Monographs volumes 1 to 42. Lyon (France): IARC; 1987. IARC monographs on the evaluation of carcinogenic risks to humans, supplement 7 .

7. Siemiatycki J, Richardson L, Straif K, Latreille B, Lakhani R, Campbell S, et al. Listing occupational carcinogens. Environ Health Perspect. 2004;112:1447-59.

8. Siemiatycki J, Dewar R, Nadon L, Gerin M. Occupational risk factors for bladder cancer: results from a case-control study in Montreal, Quebec, Canada. Am J Epidemiol. 1994;140:106180.

9. Band PR, Le ND, MacArthur AC, Fang R, Gallagher RP. Identification of occupational cancer risks in British Columbia: a population-based case-control study of bladder cancer. J Occup Environ Med. 2005;47:854-8.

10. Band PR, Spinelli JJ, Threlfall WJ, Fang R, Le ND, Gallagher RP. Identification of occupational cancer risks in British Columbia, part I: methodology, descriptive results, and analysis of cancer risks by cigarette smoking categories of 15,463 incident cancer cases. J Occup Environ Med. 1999;41:224-32.

11. World Health Organization (WHO). International classification of diseases: ninth revision. Geneva: WHO; 1977.

12. Statistics Canada. Standard industrial classification 1980. Ottawa: Minister of Supplies and Services Canada; 1981. Statistics Canada Catalogue 12-501E.

13. Statistics Canada. Standard occupational classification 1980. Ottawa: Minister of Supplies and Services Canada; 1981. Statistics Canada Catalogue 12-565E.

14. Sieber WK, Sundin DS, Frazier TM, Robinson CF. Development, use, and availability of a job exposure matrix based on national occupational hazard survey data. Am J Ind Med. 1991;20:163-74.

15. Sieber WK, Seta JA, Young RO. A job-exposure matrix for identification of potential exposures in occupational settings. In: Proceedings of the 9th International Symposium on Epidemiology in Occupational Health; September 23-25, 1992; Cincinnati, Ohio. Cincinnati $(\mathrm{OH})$ : National Institute for Occupational Safety and Health (NIOSH); 1994. p 341-6. DHHS NIOSH publication no 94-112.

16. Bureau of the Census. Alphabetical index to industries and occupations, 1980. Washington (DC): US Department of Commerce, Bureau of the Census; 1980.

17. Svirchev LM, Kan D, Lai AM, Ng V, Moody JM, Band PR. Translations of the United States industrial and occupational classifications into the Canadian standard industrial and occupational classifications. Vancouver (BC): British Columbia Cancer Agency; 1993. British Columbia Cancer Agency Monograph, number 4.

18. Breslow NE, Day NE. Statistical methods in cancer research, volume 1, the analysis of case-control studies. Lyon (France): International Agency for Research on Cancer (IARC); 1980. IARC Scientific Publication, number 32.

19. Rothman KJ, Greenland S. Modern epidemiology. 2nd edition. Philadelphia (PA): Lippincott Williams \& Wilkins; 1998.

20. Benjamini Y, Hochberg Y. Controlling the false discovery rate: a practical and powerful approach to multiple comparisons. J R Stat Soc Ser B. 1995;57:289-300.

21. Jolliffe IT. Principal component analysis. 2nd edition. New York (NY): Springer-Verlag; 2002. Springer Series in Statistics.

22. Bouyer J, Hemon D. Retrospective evaluation of occupational exposures in population-based case-control studies: general overview with special attention to job exposure matrices. Int J Epidemiol. 1993;22:S57-64.

23. Steineck G, Plato N, Alfredsson L, Norell SE. Industry-related urothelial carcinogens: application of a job-exposure matrix to census data. Am J Ind Med. 1989;16(2):209-24. 
24. Settimi L, Comba P, Bosia S, Ciapini C, Desideri E, Fedi A, et al. Cancer risk among male farmers: a multi-site case-control study. Int J Occup Med Environ Health. 2001;14:339-47.

25. Keefe AR, Bert JL, Grace JR, Makaroff SJ, Lang BJ, Band PR. A hierarchical approach to coding chemical, biological and pharmaceutical substances. J Toxicol Environ Health B Crit Rev. 2005;8(3-5):145-452.

26. Rybicki BA, Johnson CC, Peterson EL, Kortsha GX, Gorell JM. Comparability of different methods of retrospective exposure assessment of metals in manufacturing industries. Am J Ind Med. 1997;31:36-43.

27. Smith AH, Pearce NE, Callas PW. Cancer case-control studies with other cancers as controls. Int J Epidemiol. 1988;17;298306.

28. Randem BG, Burstyn I, Langard S, Svane O, Järvholm B, Kauppinen T, et al. Cancer incidence of Nordic asphalt workers. Scand J Work Environ Health. 2004;30(5):350-5.

29. Siemiatycki J. Risk factors for cancer in the workplace. Boca Raton (FL): CRC Press; 1991.

30. International Agency for Research on Cancer (IARC). Diesel and gasoline engine exhausts and some nitroarenes. Lyon (France): IARC; 1989. IARC monographs on the evaluation of carcinogenic risks to humans, number 46.

31. Pirastu R, Lavarone I, Comba P. Bladder cancer: a selected review of the epidemiological literature. Ann Inst Super Sanitá. 1996;32(1):3-20.

32. Boffetta P, Jourenkova N, Gustavsson P. Cancer risk from occupational and environmental exposure to polycyclic aromatic hydrocarbons. Cancer Causes Control. 1997;8:444-72.

33. Zeegers MPA, Swaen GMH, Kant I, Goldbohm RA, van den Brandt PA. Occupational risk factors for male bladder cancer: results from a population based case cohort study in the Netherlands. Occup Environ Med. 2001;58:590-6.

34. Vineis P, Pirastu R. Aromatic amines and cancer. Cancer Causes Control. 1997;8(3):346-55.

35. International Agency for Research on Cancer (IARC). Some non-heterocyclic polycyclic aromatic hydrocarbons and some related exposures. Lyon (France): IARC; 2005. IARC monographs on the evaluation of carcinogenic risks to humans, number 92 .

36. Ugnat AM, Luo W, Semenciw R, Mao Y. Occupational exposure to chemical and petrochemical industries and bladder cancer risk in four western Canadian provinces. Chronic Dis Can. 2004;25(2):7-15.

37. Tolbert PE. Oils and cancer. Cancer Causes Control. 1997;8:386-405.

38. Calvert GM, Ward E, Schnorr TM, Fine LJ. Cancer risks among workers exposed to metalworking fluids: a systematic review. Am J Ind Med. 1998;33:282-92.
39. Mirer F. Updated epidemiology of workers exposed to metalworking fluids provides sufficient evidence for carcinogenicity. Appl Occup Environ Hyg. 2003;18:902-12.

40. Nilsson R, Nordlinder R, Moen BE, Ovrebo S, Bleie K, Skorve AH, et al. Increased urinary excretion of 8-hydroxydeoxyguanosine in engine room personnel exposed to polycyclic aromatic hydrocarbons. Occup Environ Med. 2004;61:692-6.

41. Wartenberg D, Reyner D, Siegel Scott C. Trichloroethylene and cancer: epidemiologic evidence. Environmental Health Perspect. 2000;108(S2):161-76.

42. Teschke K, Morgan MS, Checkoway H, Franklin G, Spinelli JJ, van Belle G, et al. Surveillance of nasal and bladder cancer to locate sources of exposure to occupational carcinogens. Occup Environ Med. 1997;54:443-51.

43. Zheng T, Cantor KP, Zhang Y, Lynch CF. Occupation and bladder cancer: a population-based, case-control study in Iowa. J Occup Environ Med. 2002;44:685-91.

44. Hoove R, Fraumeni JF. Cancer Mortality in U.S. Counties with Chemical Industries. Environ Res. 1975;9:196-207.

45. International Agency for Research on Cancer (IARC). Some chemicals that cause tumours of the kidney or urinary bladder in rodents and some other substances. Lyon (France): IARC; 1999. IARC monographs on the evaluation of carcinogenic risks to humans, number 73 .

46. Iavicoli I, Carelli G. Occupational exposure to methyl tertiary butyl ether: a risk to be assessed. Occup Med (Lond). 2003;53(6):408-9.

47. Kunze E, Chang-Claude J, Frentzel-Beyme R. Life style and occupational risk factors for bladder cancer in Germany: a case-control study. Cancer. 1992;69:1776-90.

48. Guo J, Kauppinen T, Kyyrönen P, Heikkilä P, Lindbohm ML, Pukkala E. Risk of esophageal, ovarian, testicular, kidney and bladder cancers and leukemia among Finnish workers exposed to diesel or gasoline engine exhaust. Int $\mathrm{J}$ Cancer. 2004;111(2):286-92.

49. Boffetta P, Silverman DT. A meta-analysis of bladder cancer and diesel exhaust exposure. Epidemiology. 2001;12:125-30.

50. Spinelli JJ, Band PR, Svirchev LM, Gallagher RP. Mortality and cancer incidence in aluminum reduction plant workers. $\mathrm{J}$ Occup Med. 1991;33(11):1150-5.

51. International Agency for Research on Cancer (IARC). Occupational exposures of hairdressers and barbers and personal use of hair colourants; some hair dyes, cosmetic colourants, industrial dyestuffs and aromatic amines. Lyon (France): IARC; 1993. IARC monographs on the evaluation of carcinogenic risks to humans, number 57 .

Received for publication: 12 December 2006 